\title{
Laser-Cooled Atoms and Molecules Collide in a Trap
}

\author{
An experiment shows the circumstances under which ultracold atoms are \\ quick to kick molecules out of a trap, providing clues for how to use atoms \\ as a refrigerant for molecules.
}

\section{By Rachel Berkowitz}

aser cooling can chill molecules to just a few microkelvin, but some experiments demand even colder temperatures. One way that researchers propose to achieve these temperatures is to start with laser-cooled molecules, then remove even more energy via elastic collisions with ultracold atoms. However, some atom-molecule collisions are inelastic and should knock molecules out of the trap. Now, Sarunas Jurgilas and colleagues, at Imperial College London and Durham University, UK, have produced such a mixture of laser-cooled molecules and atoms and have determined the situations in which these inelastic collision losses occur [1].

The team loaded laser-cooled rubidium atoms and calcium monofluoride ( $\mathrm{CaF}$ ) molecules into a magnetic trap, then measured the number of each species remaining several seconds later. Comparing these measurements to traps holding only $\mathrm{CaF}$ molecules revealed the rate at which molecules escaped the trap as a result of interactions with rubidium

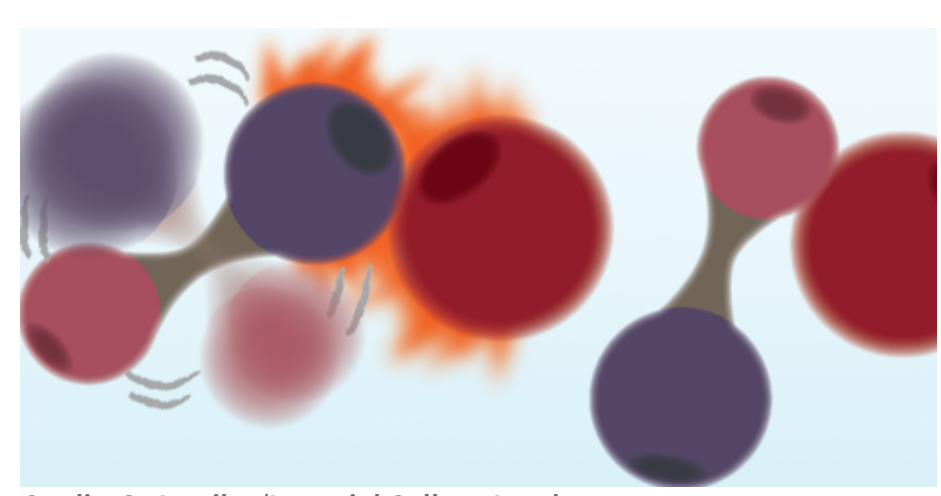

Credit: S. Jurgilas/Imperial College London atoms. Two cases illuminated the mechanism behind these losses: In one experiment, the CaF molecules started in the rotational ground state; in another, they started in a rotationally excited state. For the excited state, the loss rate corresponded to a molecule escaping every time it came close to an atom. For the ground state, the loss rate was at least 10 times smaller. The result implies that loss is due to inelastic collisions in which molecules exchange rotational energy for translational kinetic energy. Controlling molecules' rotational degrees of freedom may be key to avoiding such collisions.

For atom-molecule mixtures where elastic collisions outnumber inelastic collisions 100 -fold, collisional cooling should work well. This cooling technique could improve tests of fundamental physics that rely on precise measurements of molecular energies and could enable new capabilities in quantum computing and simulation.

Rachel Berkowitz is a Corresponding Editor for Physics based in Vancouver, Canada.

\section{REFERENCES}

1. S. Jurgilas et al., "Collisions between ultracold molecules and atoms in a magnetic trap," Phys. Rev. Lett. 126, 153401 (2021). 